\title{
Synthesis of Polyhydroxyalkanoates in Municipal Wastewater Treatment
}

\author{
Erik R. Coats ${ }^{1}$, Frank J. Loge ${ }^{2 *}$, Michael P. Wolcott ${ }^{3}$, Karl Englund ${ }^{4}$, Armando G. McDonald ${ }^{5}$
}

\begin{abstract}
Biologically derived polyesters known as polyhydroxyalkanoates (PHAs) represent a potentially "sustainable" replacement to fossilfuel-based thermoplastics. However, current commercial practices that produce PHA with pure microbial cultures grown on renewable, but refined, feedstocks (i.e., glucose) under sterile conditions do not represent a sustainable commodity. Here, we report on PHA production with a mixed microbial consortium indigenous to an activated sludge process on carbon present in municipal wastewaters. Reactors operated under anaerobic/aerobic and aerobic-only mode and fed primary solids fermenter liquor maintained a mixed microbial consortium capable of synthesizing PHA at 10 to $25 \%$ (w/w), while reducing soluble COD by approximately 62 to $71 \%$. More critically, an aerobic batch reactor seeded from the anaerobic/aerobic reactor and fed fermenter liquor achieved approximately 53\% PHA (w/w). Results presented suggest that environmentally benign production of biodegradable polymers is feasible. We further used PHA-rich biomass to produce a natural fiber-reinforced thermoplastic composite that can be used to offset advanced wastewater treatment costs. Water Environ. Res., 79, 2396 (2007).
\end{abstract}

KEYWORDS: polyhydroxyalkanoates, polyhydroxybutyrate, polyhydroxyvalerate, natural fiber-reinforced thermoplastic composites, primary solids fermenter liquor, municipal wastewater treatment.

doi: $10.2175 / 106143007 \mathrm{X} 183907$

\section{Introduction}

In our interest to move toward a more sustainable society, we strive to make human activities environmentally benign and manufacturing processes "green". We focus not only on the production of chemical commodities from renewable feedstocks, but life-cycle analyses of products and associated manufacturing processes (Marteel et. al., 2003), to ensure that, while modification of certain elements within a manufacturing process may satisfy one or more of the guiding principles of green engineering, the environmental effects have not simply shifted within the product life cycle (Anastas and Zimmerman, 2003). For example, biologically derived polyesters known as polyhydroxyalkanoates (PHAs) represent a potentially sustainable replacement to fossilfuel-based thermoplastics. Current commercial production practices

\footnotetext{
${ }^{1}$ Department of Civil Engineering, University of Idaho, Moscow, Idaho.

${ }^{2}$ Department of Civil and Environmental Engineering, University of California, Davis, California.

${ }^{3}$ Department of Civil and Environmental Engineering, Washington State University, Pullman, Washington.

${ }^{4}$ Wood Materials and Engineering Laboratory, Washington State University, Pullman, Washington.

${ }^{5}$ Department of Forest Products, University of Idaho, Moscow, Idaho.

* Department of Civil and Environmental Engineering, University of California Davis, 1 Shields Avenue, Davis, CA 95616; e-mail: fjloge@ ucdavis.edu.
}

fermentatively produce PHA with pure microbial cultures grown on renewable, but refined, feedstocks (i.e., glucose) under sterile conditions (Braunegg et. al., 2003). However, recent research has shown that the commercial production of PHA exhibits higher fossil-fuel demands and generates more carbon emissions, largely associated with feedstock production and the fermentation process, than fossil-fuel-based plastics (Gerngross, 1999). Hence, biologically derived PHA currently is not environmentally benign.

Synthesis of PHAs, which serve as bacterial carbon and energy storage reserves, is accomplished by over 300 different bacterial species, in the form of cytoplasmic granules (Lee, 1996). Biosynthesis is stimulated by either excess soluble carbon with a concurrent macronutrient limitation (typically limited on either nitrogen or phosphorus), a limitation in a terminal electron acceptor (with oxygen as the most common), or a so-called feast/famine environment, wherein microorganisms realize a transient excess of soluble carbon without any nutrient limitations (Dionisi, Majone, Papa, and Beccari, 2004). Poly-3-hydroxybutyrate (PHB or P3HB) was the first PHA discovered (over 75 years ago) and hence is the most extensively characterized type (Lemoigne, 1926; Madison and Huisman, 1999). Since this initial discovery, many forms of hydroxyalkanoic monomer units have been identified (Madison and Huisman, 1999). Common precursors to PHA synthesis include simple sugars, such as glucose and fructose, and organic acids, such as acetic and propionic acid. The type of carbon substrate dictates the polymeric structure of the PHA (Madison and Huisman, 1999), with some of the most commonly studied forms including PHB, polyhydroxyvalerate (PHV), and poly-4-hydroxybutyrate (P4HB). In turn, each form of PHA yields different polymer properties. The PHB exhibits similar properties to polypropylene, including melting temperature and crystallinity, but the polymer is brittle upon crystallization and exhibits little stress resistance (Madison and Huisman, 1999). Polymer improvements have been accomplished through copolymerization with $\mathrm{PHV}$, to increase ductility and impact resistance and lower processing temperatures (Madison and Huisman, 1999).

With current biological PHA production practices (Braunegg et al., 2003), the feedstock is estimated to account for approximately 30 to $50 \%$ of the total production cost and approximately 12 to $33 \%$ of the total energy requirements (Akiyama et al., 2003; Choi and Lee, 1997; Gerngross, 1999). These estimates vary somewhat with the microbial species used, carbon source, PHA yield, and PHA production capacity (Akiyama et al., 2003; Choi and Lee, 1997; Gerngross, 1999). Bioreactor operations used to produce PHA consume an additional approximately 30 to $40 \%$ in energy (Akiyama et al., 2003; Gerngross, 1999), with a concomitant share of the product cost. Research to minimize these energy and cost elements has focused on either the use of pure cultures with waste 
streams rich in precursor carbohydrates and organic acids (Ashby et al., 2004; Hassan et al., 2002; Kahar et al., 2004; Yu and Si, 2001), pure microbial cultures with waste streams derived from refined waste feedstocks ( $\mathrm{Du}$ and $\mathrm{Yu}, 2002$ ), or mixed microbial consortia grown on synthetic feedstocks (Beun et al., 2000, 2002; Dionisi, Majone, Papa, and Beccari, 2004; Dionisi, Renzi, Majone, Beccari, and Ramadori, 2004; Takabatake et. al., 2000). The concept of producing commercial quantities of PHA, with wild microbial consortia using, as feedstock, refined solid or liquid wastes, has been proposed (Majone et al., 1999; Reis et al., 2003), but never tested. Furthermore, integration with and interconnectivity to raw material flow (Anastas and Zimmerman, 2003), wherein commercial production of PHA occurs within a wastewater treatment system using influent wastewater as the feedstock, has neither been proposed nor tested. Considering the apparent propensity for wild microbial consortia to synthesize the polymer (Beun et al., 2002; Carucci et al., 2001; Dionisi, Renzi, Majone, Beccari, and Ramadori, 2004), commercial production of PHA would theoretically appear to be a natural extension of the biological treatment regime; in fact, PHA synthesis is empirically associated with certain municipal wastewater treatment processes (Comeau et al., 1986; Mino et al., 1987; Randall and Liu, 2002). However, biological synthesis of PHA in full-scale wastewater treatment facilities, estimated at upwards of $4 \%$ (w/w) (data not shown), falls short of quantities necessary for commercial exploitation. Nevertheless, recognizing that many waste streams are rich in PHA precursors, the potential for process integration exists.

The goal of this research was to demonstrate that commercial PHA production could be integrated into various configurations of municipal wastewater treatment schemes and that conditions could be established to concurrently achieve treatment objectives and generate high PHA yields. The specific objectives were to

(1) Identify suitable municipal waste streams for the production of PHA;

(2) Evaluate coupled PHA production and wastewater treatment within laboratory-based bioreactors; and

(3) Demonstrate that PHB, in an unpurified form, can be used in the production of common commercial products, thereby reducing the polymer processing costs.

\section{Materials and Methods}

Source of Microorganisms. The mixed microbial seed was obtained from the Moscow, Idaho, wastewater treatment facility, which had been determined, through a series of preliminary facility screening studies (data not shown), to be capable of synthesizing PHA. The microbial seed for the primary solids fermenter was obtained from the anaerobic digester at the Pullman, Washington, wastewater treatment plant. Azotobacter vinelandii UWD was purchased from the American Type Culture Collection (ATCC 53799).

Source of Wastewater. Raw wastewater was obtained from the Moscow, Idaho wastewater treatment facility. Thickened primary solids, for operation of the fermenter, were obtained from the Pullman, Washington, wastewater treatment facility.

Culture Conditions, Growth Media, and Harvesting Procedures. Fermentate was produced in a $10-\mathrm{L}$ completely mixed primary solids fermenter operated as a sequencing batch reactor (SBR) with a 24-hour reaction time and a 4-day solids retention time (SRT). The fill and withdraw cycles of the SBR occurred almost immediately. Given that the solution under reaction in the
SBR was not permitted to settle before withdrawal, the hydraulic retention time (HRT) was equivalent to the SRT. The daily decant was centrifuged at approximately $10000 \times \mathrm{g}$ and the supernatant (i.e., fermentate) recovered. The fermentate-fed anaerobic/aerobic reactor and the raw wastewater and raw wastewater-methanol fed reactors, consisted of 4-L SBRs continuously operated on a 24-hour reaction cycle (anaerobically for 6 hours following feeding, then aerobically for 18 hours), with an SRT and HRT of 5 days. The fermenter-fed aerobic PHA-wastewater treatment reactor was operated in a similar manner, except that the reactor was aerated continuously over the 24-hour reaction period. Anaerobic conditions were accomplished through the continuous supply of nitrogen gas, while aerobic conditions were maintained through the supply of air. Nitrogen gas and air were supplied through a 23-cm- (9-in.-) diameter Sanitaire Silver Series II membrane fine-bubble disc diffuser (Sanitaire, Brown Deer, Wisconsin) installed at the base of the reactor. Dissolved oxygen was monitored, to verify that anaerobic and aerobic conditions were achieved. The reactors were operated at room temperature (approximately $18^{\circ} \mathrm{C}\left[65^{\circ} \mathrm{F}\right]$ ).

Sidestream PHA production was accomplished in a 2 -L flask seeded, with $400 \mathrm{~mL}$ from the wastewater treatment reactor, and fed $600 \mathrm{~mL}$ of fermentate. The reactor was operated for 7 hours, continuously aerated and mixed, with a dissolved oxygen concentration in excess of $6 \mathrm{mg} / \mathrm{L}$. The reactor was operated at room temperature (approximately $18^{\circ} \mathrm{C}\left[65^{\circ} \mathrm{F}\right]$ ). The PHA-rich biomass from all wastewater-based processes was centrifuged at approximately $10000 \times \mathrm{g}$; the biomass pellet was chlorinated with $6.25 \%$ sodium hypochlorite for approximately 5 minutes, resuspended in deionized water and recentrifuged, and dried at $60^{\circ} \mathrm{C}$.

A. vinelandii UWD was cultured in $10-\mathrm{L}$ reactors aerated through a 23-cm- (9-in.-) diameter Sanitaire Silver Series II membrane fine-bubble disc diffuser. Reactors were operated continuously in two 9-month periods, for the sole purpose of producing quantities of PHB-rich biomass. The reactors were operated as SBRs with a 24hour reaction time and an SRT and HRT ranging from 1.1 to 2 days. The mixed liquor suspended solids (MLSS) concentration stabilized at approximately $5 \mathrm{~g} / \mathrm{L}$ in all reactors. The reactors were operated within environmentally controlled rooms, and the temperature was maintained at $30^{\circ} \mathrm{C}$. The medium for PHB production consisted of the following (g/L, reagent-grade): $\mathrm{KH}_{2} \mathrm{PO}_{4}, 0.16 ; \mathrm{K}_{2} \mathrm{HPO}_{4}, 0.64$; $\mathrm{MgSO}_{4} \cdot 7 \mathrm{H}_{2} \mathrm{O}, 0.16 ; \mathrm{CaSO}_{4} \cdot 2 \mathrm{H}_{2} \mathrm{O}, 0.08 ; \mathrm{FeCl}_{3}, 0.04 ; \mathrm{Na}_{2-}$ $\mathrm{MoO}_{4} \cdot 2 \mathrm{H}_{2} \mathrm{O}$, 0.008; glucose, 20; and sucrose, 20. The medium was autoclaved before addition to the reactors. Daily reactor decants of PHB-rich biomass were processed as specified previously.

Analytical Techniques. Soluble chemical oxygen demand (sCOD or COD) tests were performed in accordance with Standard Methods 5220-D (APHA et al., 1998), with samples filtered through sterilized $0.22-\mu \mathrm{m}$ filters before analysis (Millipore Corporation, Billerica, Massachusetts) in Hach high-range ampules (Hach Company, Loveland, Colorado), with a Hach COD reactor and a Spectronic 20 Genesys spectrophotometer (Thermo Electron Corporation. Waltham, Massachusetts). The MLSS concentration was determined gravimetrically, in accordance with Standard Methods 2540-D (APHA et al., 1998), with Millipore 1.2- $\mu \mathrm{m}$ glass/fiber filters (Millipore Corporation). Organic carbon constituents in the fermentate were determined through ion exclusion high performance liquid chromatography (HPLC) analysis, using a Rezex ROA organic acid column (Phenomenex, Torrance, California) and a Waters HPLC (Waters, Milford, Massachusetts) equipped with both UV $(210 \mathrm{~nm})$ and differential refractive index detectors. Aqueous sulfuric acid $(0.005 \mathrm{~N})$ was used as the mobile phase, and 
separations were carried out at $65^{\circ} \mathrm{C}$ at a flowrate of $0.5 \mathrm{~mL} / \mathrm{min}$. Biomass PHA content was determined by gas chromatography/mass spectrometry (GC-MS) (Braunegg et al., 1978). Briefly, dried PHArich biomass samples were digested at $100^{\circ} \mathrm{C}$ in $2 \mathrm{~mL}$ each of acidified methanol ( $3 \% \mathrm{v} / \mathrm{v}$ sulfuric acid) and chloroform. Benzoic acid was added to the chloroform as an internal standard. Following vigorous vortexing of the mixture with $1 \mathrm{~mL}$ deionized water, PHArich chloroform was recovered for analysis. The chloroform phase was dehydrated by filtering the PHA-rich solution through sodium sulfate before analysis. The GC-MS was performed on a Thermofinnigan PolarisQ iontrap GC-MS instrument (Thermo Electron Corporation) in positive ei mode. The sample was introduced using split injection. Separation was achieved on a ZB1 (15 m, 0.25-mm internal diameter) capillary column (Phenomenex), with helium as the carrier gas $(1.2 \mathrm{~mL} / \mathrm{min})$ and an initial temperature of $40^{\circ} \mathrm{C}$ ( 2 minutes) ramped to $200^{\circ} \mathrm{C}$ at $5^{\circ} \mathrm{C} / \mathrm{min}$. The Xcalibur software program (Thermo Electron Corporation) was used to analyze the data. The compounds were confirmed by retention time and mass spectral matching with known standards (Lancaster Synthesis, Ward Hill, Massachusetts) as methyl ester derivatives and quantified based on the internal standard. Total cellular PHA content was determined on a percent dry weight cell basis (i.e., mass PHA/mass of biomass, w/w).

Composite Processing. Natural fiber-reinforced thermoplastic composites (NFRTCs) were manufactured with PHB-rich biomass, purified P3HB obtained from CarboMer (San Diego, California), and polypropylene obtained from Solvay (BP Solvay, Houston, Texas). Sixty-mesh wood flour was obtained from American Wood Fibers (Schofield, Wisconsin) and added to the composite formulation as the natural fiber reinforcement. Dry materials were melt-blended in a torque rheometer for 5 minutes at $170^{\circ} \mathrm{C}$, then fed into a capillary rheometer with a barrel temperature of $170^{\circ} \mathrm{C}$, and finally injected to a heated die creating two solid rectangular bars $(5 \mathrm{~mm} \times 12 \mathrm{~mm} \times 94 \mathrm{~mm})$. Once the bars were removed from the die, they were allowed to equilibrate for 1 week at $22^{\circ} \mathrm{C}$ and $50 \%$ relative humidity. The conditioned specimens were then tested in flexure, according to the ASTM D790 procedures (ASTM, 1997). The stiffness (modulus of elasticity [MOE]), bending strength (modulus of rupture $[\mathrm{MOR}]$ ), and strain at break were calculated and reported.

\section{Results and Discussion}

Screening of Waste Streams. Municipal wastewater streams are typically carbon-limited and thus would generally not be suitable for excess PHA production. However, some municipal treatment facilities incorporate primary solids fermentation to generate a waste stream rich in soluble short-chain organic acids (principally acetate) necessary to elicit certain target metabolic responses (Barnard and Scruggs, 2003). Previous research has suggested that a wild microbial consortium fed such a carbon-rich, nutrient-balanced substrate will overproduce PHA (Dionisi, Majone, Papa, and Beccari, 2004). Hence, fermentate was considered a candidate waste stream for commercial PHA production in municipal wastewater treatment.

A laboratory-based fermenter was used to generate the required waste stream. The fermenter was operated at an SRT of 4 days, consistent with recommended design guidelines (Grady et al., 1999), and batch-fed primary solids at a concentration of 3 to $4 \%$ $(\mathrm{w} / \mathrm{v})$. Fermentate exhibited a sCOD ranging from approximately 1500 to $1700 \mathrm{mg} / \mathrm{L}$. The HPLC analyses confirmed that the primary carbon constituents were acetic acid and propionic acid, at

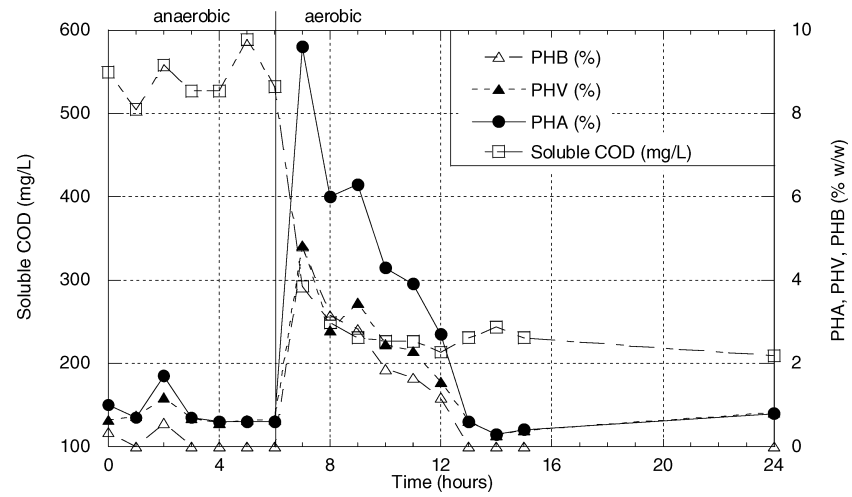

Figure 1-Soluble COD, PHAs, PHB, and PHV in an anaerobic/aerobic SBR seeded with a mixed microbial consortium and fed fermentate (at $t=0$ ) derived from municipal primary solids (December 17, 2004 sampling and analysis date).

concentrations of approximately 825 and $250 \mathrm{mg} / \mathrm{L}$, respectively. The fermentate was evaluated against standards for acetic, propionic, butyric, pentanoic acid, ethanol, and methanol. A nominal amount of the larger chain carboxylic acids also appeared to be synthesized, with no significant alcohol products. Applying typical stoichiometric ratios for acetic and propionic acid (i.e., milligrams COD:milligrams acid) (Grady et al., 1999), these two constituents were confirmed to be the predominant forms of readily biodegradable carbon in the fermentate.

Methanol augmentation to carbon-limited raw wastewater was selected as a second candidate wastewater. Methanol was chosen because

(1) The carbon concentration could be directly increased without altering the other raw wastewater nutrient concentrations, thus yielding a nutrient imbalanced growth media.

(2) This form of carbon would provide a contrast with the carboxylic acid-rich fermenter liquor.

(3) Methanol is used at full-scale wastewater treatment facilities to enhance denitrification; hence, it is a carbon source associated with full-scale wastewater treatment (Tchobanoglous et al., 2003).

(4) Methanol is a direct precursor to PHB synthesis (Ackermann and Wolfgang, 1998; Yamane, 1993).

Polyhydroxyalkanoate Production within an Anaerobic/ Aerobic Wastewater Treatment Environment. Using a PHAproducing mixed microbial seed obtained from the Moscow, Idaho, wastewater treatment facility, a fermentate-fed SBR operated in an alternating anaerobic/aerobic scheme consistently maintained a microbial consortium capable of producing relatively significant quantities of PHA, while concurrently reducing SCOD by approximately $62 \%$ (Figure 1). The results presented in Figure 1 were collected on day 32 of reactor operations. The reactor was operated with an SRT and HRT of 5 days and a 24-hour operational cycle; the $\mathrm{pH}$ varied between 8 and 9. The concentration of sCOD remained approximately constant over the 6-hour anaerobic phase, then declined at a first-order rate aerobically. The PHA synthesis occurred principally during the aerobic period and followed a feastfamine pattern (i.e., rapid carbon use concurrent with rapid PHA synthesis, followed by rapid PHA depletion); maximum PHA production consistently occurred at a timepoint concurrent with the 


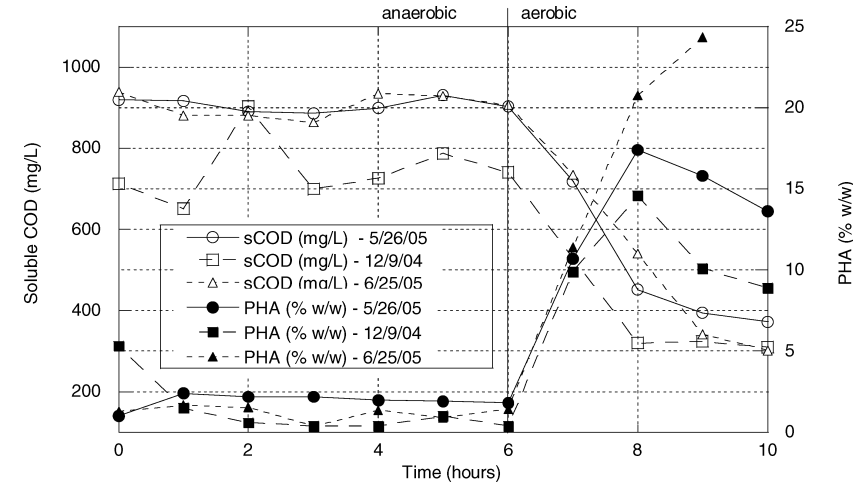

Figure 2-Soluble COD and PHAs in an anaerobic/aerobic SBR. Data represents results from reactor operations from two independent reactor operations (November/ December 2004 and May/June 2005). The June 25, 2005, results represent reactor operations switched from raw wastewater/methanol (i.e., Figure 5) to fermentate (fed at $t=0$ ) derived from municipal primary solids.

maximum reduction in readily metabolized soluble carbon. Importantly, co-polymerization of both PHB and PHV was achieved (Figure 1). The occurrence of this feast-famine pattern is consistent with previous investigations (Beun et al., 2002; Carucci et al., 2001; Dionisi, Majone, Papa, and Beccari, 2004), which focused on environmental matrices other than wild microbial consortia and wastewater.

The described conditions were replicated in three discrete reactors operated under steady-state conditions at different times over a 9-month period. Comparable wastewater treatment efficiency and PHA production patterns were achieved on a recurring basis for each discrete reactor (Figure 2). While peak PHA production did vary temporally and between reactors, with a range from approximately 10 to $25 \%$, the feast-famine pattern was consistently replicated and, most critical for process success, the microbial consortium consistently maintained the ability to produce PHA. Each of these reactors was established with a new microbial seed obtained from the Moscow, Idaho, facility, with the primary solids fermenter operated with similarly new (i.e., "fresh") material. Further, the reactor microbial seed and primary solids were obtained

Table 1-PHA yield during the carbon 'feast' period within the anaerobic/aerobic wastewater treatment reactors operated May through June 2005.

\begin{tabular}{|c|c|c|c|c|}
\hline Sample date & $\begin{array}{l}\text { MLSS } \\
\text { (mg/L) }\end{array}$ & $\begin{array}{c}\text { Substrate } \\
\text { consumed }^{\mathrm{a}} \\
\text { (mg sCOD/L) }\end{array}$ & $\begin{array}{c}\text { PHA } \\
\text { synthesized } \\
\text { (mg COD/L) }\end{array}$ & $\begin{array}{l}\text { PHA yield } \\
\text { (mg COD/mg } \\
\text { sCOD) }\end{array}$ \\
\hline May 26, 2005 & 1920 & 478 & 456 & 0.95 \\
\hline June 25, 2005 & 1500 & 597 & 520 & 0.87 \\
\hline
\end{tabular}

a Substrate consumed and PHA synthesized were calculated during the carbon "feast" period to the timepoint of peak PHA cellular concentration, which, for each sampling event, was 3 and 2 hours, respectively. The mass of PHA was converted to a COD basis using stoichiometric ratios for PHB and PHV

b PHA yield was calculated as PHA synthesized divided by substrate consumed.

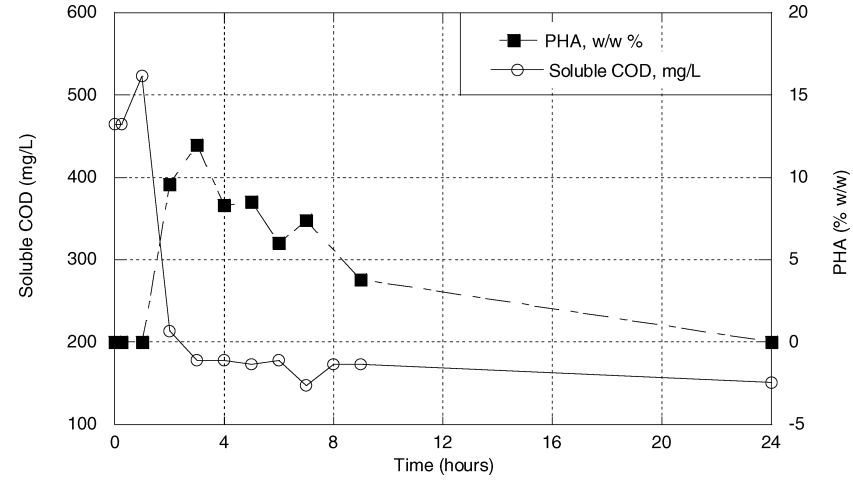

Figure 3-Soluble COD and PHAs in an aerobic SBR seeded with a mixed microbial consortium and fed fermentate (at $t=0$ ) derived from municipal primary solids.

under different seasonal conditions (i.e., fall, winter, and spring) and under varying city wastewater conditions (i.e., with and without the large university student populations in Pullman and Moscow). Thus, in our view, the results represent a robust and stable operating condition.

Beyond the stability of the proposed process, another observation carries perhaps equivalent weight. Using stoichiometric ratios (milligrams COD:milligrams PHA) for PHB and PHV of 1.38 and 1.63 (Dionisi, Majone, Papa, and Beccari, 2004), the PHA storage yield values (milligrams COD PHA synthesized per milligram sCOD substrate removed) for cycles measured on May 26 and June 25, 2005, were 0.95 and 0.87, respectively (Table 1). These results validate that a carbon "feast" condition occurred immediately within the aerobic period, with most of the carbon shunted to PHA. Others have made similar determinations with mixed microbial consortia and synthetic organic acid substrates (Dionisi, Majone, Papa, and Beccari, 2004; Dionisi, Renzi, Majone, Beccari, and Ramadori, 2004).

Polyhydroxyalkanoate Production within an Aerobic Wastewater Treatment Environment. Using a PHA-producing mixed microbial seed from the Moscow, Idaho, wastewater treatment facility, a fermentate-fed aerobic SBR concurrently achieved carbon removal and PHA synthesis (Figure 3), on a recurring basis over a 32-day period. This reactor was operated as a carbon removal activated sludge wastewater treatment system (Tchobanoglous et al., 2003), with an SRT and HRT of 5 days and a 24-hour operational cycle; the $\mathrm{pH}$ varied between 8 and 9. The sCOD concentrations diminished simultaneously, according to first-order reaction rate kinetics. Steady-state concentrations were effectively achieved within 8 hours, with $67 \%$ sCOD removal; over 24 hours, approximately $71 \%$ sCOD removal was achieved. The PHA synthesis occurred concurrently with SCOD depletion, peaking with the depletion of substrate (Figure 3). The PHA reserves were ultimately depleted within the 24-hour cycle. The PHA synthesis and sCOD depletion patterns were effectively identical to those observed in the anaerobic/aerobic wastewater treatment reactor, with PHA synthesis again following a feast-famine pattern.

Excess Polyhydroxyalkanoate Production in a Sidestream Reactor. The fermentate-fed wastewater treatment reactors maintained a microbial consortium capable of overproducing PHA on a consistent and reliable basis; however, the quantity of PHA was not at a commercial level. To further increase the concentration of 


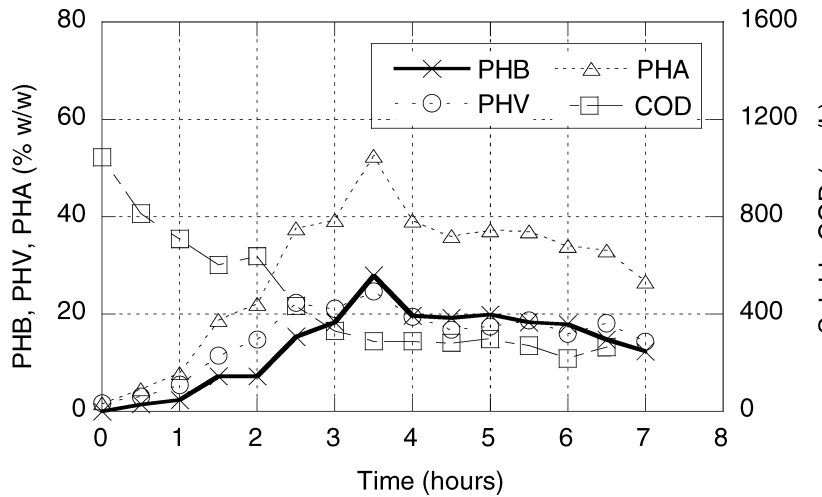

Figure 4-Soluble COD, PHAs, PHB, and PHV in a sidestream aerobic batch reactor seeded with cells obtained from the SBR in Figure 1 at $t=24$ hours and fed fermentate (at $t=0$ ) derived from municipal primary solids.

PHA in the biomass, fermentate was fed to a single-run, sidestream reactor seeded with biomass wasted from the anaerobic/aerobic wastewater treatment reactor at the end of a cycle. The seed concentration of biomass in the sidestream reactor, before the addition of fermentate, was approximately $1500 \mathrm{mg} / \mathrm{L}$. The volumetric ratio of fermentate-to-microbial seed was 1.5:1, compared with 0.2:1 in the treatment reactors; a higher PHA yield was thus anticipated. Within 3.5 hours, PHA production peaked, at approximately $53 \%(\mathrm{w} / \mathrm{w})$ (Figure 4). Similar results were determined with a microbial seed derived from the aerobic wastewater treatment reactor. Importantly, PHA production occurred rapidly, compared with conventional pure culture-based operations (Akiyama et al., 2003), which exceed typical HRTs used in wastewater treatment. Perhaps more critically, at least in regard to polymer mechanical properties, the PHB:PHV ratio was approximately 1:1 (Figure 4). Regarding PHA yield, considering that the carbon loading was relatively low (less than $1200 \mathrm{mg} / \mathrm{L}$ sCOD) and PHA synthesis peaked with the apparent depletion of readily metabolized carbon, these results suggest that saturation of PHA synthesis did not occur and that higher PHA yields would be achievable. Finally, recognizing that the relatively short time to peak PHA synthesis (i.e., 3.5 hours) was not sufficient for significant reproduction of PHA-producing microbes, these results strongly suggest that the microbial seed derived from the wastewater treatment reactor was highly enriched for the target microorganisms.

Polyhydroxyalkanoate Production on a Carbon-Rich, Nutrient-Poor Municipal Wastewater. Using a mixed microbial seed obtained from the Moscow, Idaho, wastewater treatment facility, two reactors were operated in an alternating anaerobic/ aerobic scheme, with one fed raw wastewater and the second fed raw wastewater supplemented with methanol $(5 \mathrm{~mL}$ methanol, yielding $0.125 \%[\mathrm{v} / \mathrm{v}]$ initial concentration). Both reactors were operated as an SBR, with an SRT and HRT of 5 days and a 24-hour reaction cycle. The reactor fed raw wastewater was ultimately shut down, as the carbon concentration was insufficient (approximately $100 \mathrm{mg} / \mathrm{L}$ as soluble COD) to maintain an actively growing microbial consortium under the established operating conditions. No operational difficulties were experienced with the reactor fed raw wastewater supplemented with methanol.

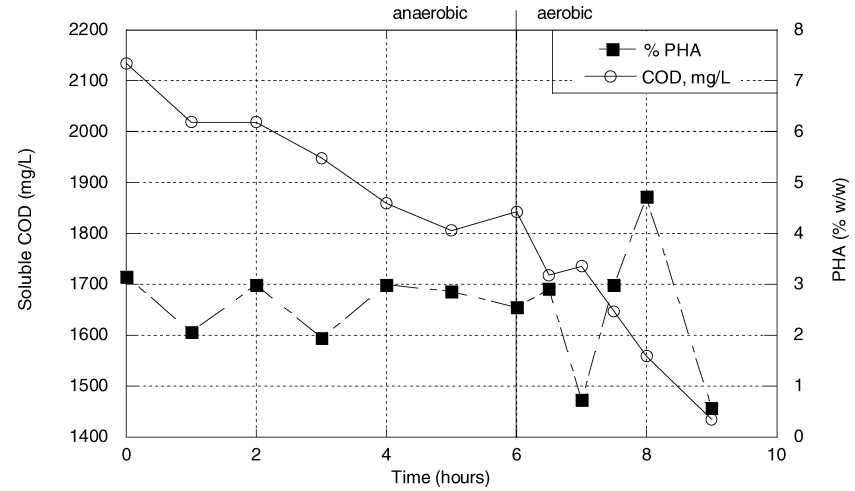

Figure 5-Soluble COD and PHAs in an anaerobic/aerobic SBR seeded with a mixed microbial consortium and fed raw wastewater and methanol (at $t=0)$.

As a contrast to the fermentate-fed wastewater treatment reactor, the consortium in the methanol-fed reactor generally used carbon at a constant rate over time throughout the anaerobic and aerobic periods; however, no appreciable PHA synthesis occurred (Figure 5). As expected given the predominate form of carbon, the only form of PHA detected was PHB. Total carbon removal during the first 9 hours was approximately 29\%, with approximately $61 \%$ removed over the full 24-hour reaction cycle; the gas-stripping rate of methanol was less than $1 \%$ /day (data not shown); hence, the observed removal was predominately attributed to biological use. Clearly, augmentation with a single carbon molecule, such as methanol, which would target a smaller candidate population of PHA-producing microbes (in this case, methylotrophs), does not yield conditions suitable for concurrent commercial PHA production and wastewater treatment. These results also support the need to achieve feast-famine conditions over nutrient deficiency as PHA synthesis stimuli.

To further evaluate the robustness of the wild microbial consortium initially fed raw wastewater supplemented with methanol, the substrate was switched to fermenter liquor. Two interesting responses were observed. First, within 2 weeks, the microbial consortium ultimately switched metabolic responses to accomplish wastewater treatment and PHA production consistent with the fermentate feedstock (Figure 2). In fact, peak PHA production approached $25 \%(\mathrm{w} / \mathrm{w})$. Second, the microbial consortium adapted to synthesize both PHB and PHV in copious quantities, whereas the consortium was previously producing only minimal amounts of PHB. Interestingly, a contrast of the respective microbial populations revealed little apparent similarity, despite receiving the same substrate (Coats, Loge, Smith, Thompson, and Wolcott, 2007).

Biological Considerations in Full-Scale Wastewater Facilities. While the simple fact that such copious amounts of PHA could be produced with an indigenous microbial consortium cultured on real wastewater certainly is significant, another observation carries similar weight. Quantitative PHA analysis on the original microbial seed indicated approximately $0.2 \%(\mathrm{w} / \mathrm{w})$ PHB and insignificant amounts of PHV, suggesting either limited numbers of PHA-producing organisms or a limited production capacity. In either case, when exposed to more optimum PHAproducing conditions, this same microbial seed flourished.

In a survey conducted as part of this study, the concentration of PHA in the MLSS collected from 10 wastewater treatment facilities ranged from 0.1 to $4 \%$ (data not shown). Given that we produced 


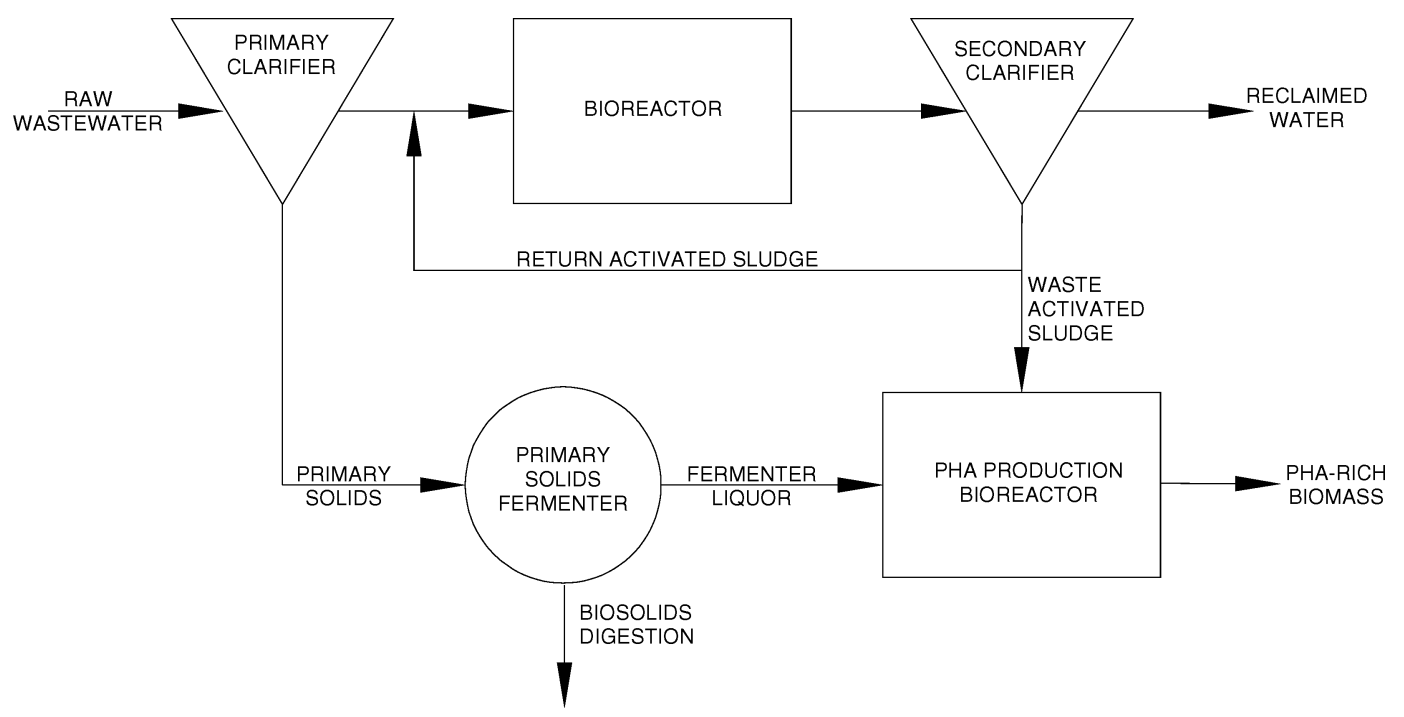

Figure 6-Schematic diagram of the proposed PHA production process integrated within a municipal wastewater treatment scheme.

commercial-level quantities of PHA from a seed culture that exhibited measurable, yet minimal, PHA content, we are assuming that the concepts presented in this paper would thus extend to all wastewater treatment facilities, with no modification to the existing microbial consortium. Further, given that all facilities evaluated as part of this study exhibited microbial PHA synthesis, we believe that modification of any suspended-growth process (i.e., conventional activated sludge) could be achieved to naturally select for a consortium capable of producing quantities of PHA that could be further enhanced with operational modifications similar to the ones implemented in this study.

Proposed Polyhydroxyalkanoate-Wastewater Treatment Scheme. Based on the results presented above, the following concept is proposed for coupled PHA production and wastewater treatment in municipal environments (Figure 6). Treatment of raw wastewater would occur in a biological treatment train consisting of reactors in series designed to create selective environmental pressures necessary to achieve removal of the target nutrients. Mass production of PHA would occur in a separate biological reactor receiving biomass routinely wasted from the wastewater treatment reactor. Primary solids fermentate, derived from a primary solids fermentation reactor, would be supplied to both the wastewater treatment reactor and PHA production reactor. The supply of fermentate (or a comparable carbon-rich, nutrient-rich waste stream) is critical to the success of this process scheme, as it provides the quantity and quality of necessary nutrients to elicit the feast-famine PHA response.

Manufacture of Natural Fiber-Reinforced Thermoplastic Composites. The commercial quantities of PHA produced within wastewater treatment could potentially be used in a range of applications spanning interior automotive components to utensils. In our view, NFRTCs represent the most immediate potential application. The NFRTCs are primarily composed of natural fibers (i.e., wood, wheat straw, and hemp) that are mixed and then heated with thermoplastics with melting temperatures less than the thermal degradation point of the natural fiber (i.e., $250^{\circ} \mathrm{C}$ for wood). Commonly used thermoplastics include poly(propylene), high- and low-density poly-(ethylene), poly(vinyl chloride), and poly(styrene). There is growing interest in the NFRTC industry to replace petroleum-based thermoplastics with biologically derived PHA. To date, attention has focused exclusively on purified PHA that is extracted and then separated from the cell debris before mixing with the natural fiber.

As a demonstration of the potential application of PHA commercially produced within a wastewater treatment plant, we mixed PHB-rich biomass, derived from Azotobacter vinelandii UWD (Page and Knosp, 1989), without purification or extraction, with wood flour. The pure culture was used as a surrogate for wastewater biomass because of the lack of odor-control equipment in the composite-manufacturing facility. The extruded NFRTCs exhibited material properties statistically similar to materials produced with purified PHB (Tables 1 and 2). Interestingly, the microbial cell debris appeared to convey material processing advantages, in that significantly lower polymer content composites could be produced using the PHB-rich biomass relative to the pure PHB NFRTCs (Coats, Loge, Wolcott, Englund, and McDonald, 2007). This is important because, arguably, there are applications even for the lower PHB-rich biomass formulations produced in this study. With further research, the brittle behavior (Table 1) of PHB NFRTCs could be alleviated, if necessary, with addition of PHV as a copolymer (Reinsch and Kelley, 1997; Shanks et al., 2004).

\section{Conclusions}

Based on the results presented herein, the following conclusions can be drawn:

(1) Commercial production of PHA could feasibly be integrated into wastewater treatment systems. The quantity of PHA that could be generated would principally be a function of the SRT, HRT, influent flowrate, influent total suspended solids, MLSS concentration, and primary solids fermenter operations. Optimization on a case-by-case basis would be necessary for process success.

(2) The proposed PHA production technology eliminates costs associated with feedstock production and has negligible energy requirements, given that wastewater treatment is mandated in most countries. 
Table 2-Preliminary material properties of resin transfer molded NFRTCs. ${ }^{a}$

Thermoplastic type

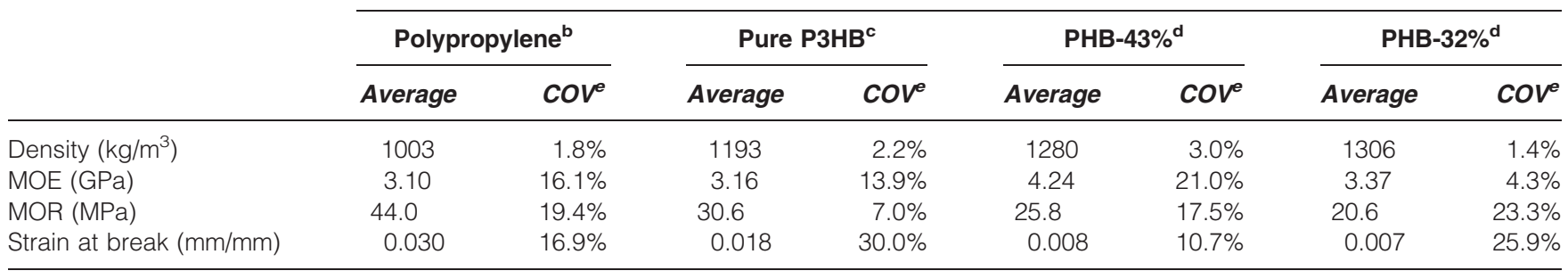

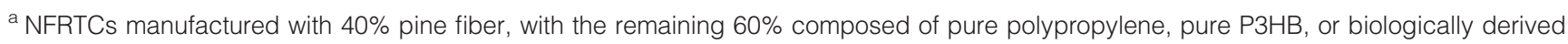
PHA plus cell debris.

${ }^{b}$ Polypropylene obtained from Solvay, BP Solvay, Houston, Texas.

${ }^{c}$ Pure P3HB obtained from CarboMer, San Diego, California.

a PHB produced biologically in Azotobacter vinelandii UWD (ATCC 53799). Percentages represent the weight of PHB on a dry microbial cell basis (not wood fiber basis). The NFRTC manufactured with PHB-43\% contained $40 \%$ pine fiber, $26 \%$ PHB $\left(0.43^{*} 0.60\right)$, and $34 \%$ cell debris; the NFRTC manufactured with PHB-32\% contained $40 \%$ pine fiber, $19 \%$ PHB $(0.32 * 0.6)$, and $41 \%$ cell debris.

${ }^{\mathrm{e}} \mathrm{COV}=$ coefficient of variation.

(3) Many existing wastewater treatment plants derive methane, and subsequently energy, through anaerobic digestion of primary and secondary solids. In the proposed PHA production technology, PHA-rich secondary solids would be diverted from the digestor and reused in some manner, such as in the manufacture of an NFRTC. Recognizing that residual primary solids from the fermenter would still require digestion, the proposed process would not necessarily replace energy generation with methane produced in an anaerobic digestor; rather, the PHA production technology represents another value-added use of organic carbon in wastewater.

(4) Use of unextracted PHA in the production of a NFRTC not only eliminates the energy and costs associated with extraction in conventional PHA production (approximately 30 to $40 \%$ of overall values) (Akiyama et al., 2003; Gerngross, 1999), but represents an innovative method for sludge disposal.

(5) The NFRTCs, produced from biologically derived PHA and biosolids, represent a commercial commodity that can be used to offset the cost of advanced wastewater treatment. Recognizing that secondary commodities associated with waste treatment processes, such as reclaimed water for nonpotable uses and biosolids used as agrofertilizers, are gaining favor, the extension to PHA production is a natural progression in the evolution beyond simply managing waste.

(6) Further studies are necessary to advance this novel polymer production process and sludge disposal method, including a detailed life-cycle analysis, development of fundamental relationships between carbon sources present in wastewaters and the type of PHA, characterization of the mixed-microbial consortia, and process optimization on the full scale.

\section{Credits}

This material is based on work supported by the National Science Foundation (Arlington, Virginia) under grant number DMI0400337. Any opinions, findings, and conclusions or recommendations expressed in this material are those of the authors and do not necessarily reflect the views of the funding agency.

Submitted for publication October 5, 2006; revised manuscript submitted March 16, 2007; accepted for publication March 20, 2007.
The deadline to submit Discussions of this paper is February 15 , 2008.

\section{References}

Ackermann, J.; Wolfgang, B. (1998) Approaches to Increase the Economy of the PHB Production. Polym. Degrad. Stabil., 59, 183-186.

Akiyama, M.; Tsuge, T.; Doi, Y. (2003) Environmental Life Cycle Comparison of Polyhydroxyalkanoates Produced from Renewable Carbon Resources by Bacterial Fermentation. Polym. Degrad. Stabil., 80, 183-194.

American Public Health Association; American Water Works Association; Water Environment Federation (1998) Standard Methods for the Examination of Water and Wastewater, 20th ed.; American Public Health Association: Washington, D.C.

Anastas, P. T.; Zimmerman, J. B. (2003) Design Through the 12 Principles of Green Engineering. Environ. Sci. Technol., 37, 94A-101A.

Ashby, R. D.; Solaiman, D. K. Y.; Foglia, T. A. (2004) Bacterial Poly(hydroxyalkanoate) Polymer Production from the Biodiesel CoProduct Stream. J. Polym. Environ., 12, 105-112.

ASTM (1997) Flexural Properties of Unreinforced and Reinforced Plastics and Electrical Insulating Materials (ASTM D790). American Society for Testing and Materials: West Conshohocken, Pennsylvania.

Barnard, J. L.; Scruggs, C. E. (2003) Biological Phosphorus Removal. Water Environ. Technol., 15, 27-33.

Beun, J. J.; Dircks, K.; Van Loosdrecht, M. C. M.; Heijnen, J. J. (2002). Poly-[beta]-hydroxybutyrate Metabolism in Dynamically Fed Mixed Microbial Cultures. Water Res., 36, 1167-1180.

Beun, J. J.; Paletta, F.; van Loosdrecht, M. C. M.; Heijnen, J. J. (2000) Stoichiometry and Kinetics of Poly-Beta-Hydroxybutyrate Metabolism in Aerobic, Slow Growing, Activated Sludge Cultures. Biotechnol. Bioeng., 67, 379-389.

Braunegg, G.; Lefebvre, G.; Genser, K. (2003) Polyhydroxyalkanoates, Biopolyesters from Renewable Resources: Physiological and Engineering Aspects. J. Biotechnol., 65, 127-161.

Braunegg, G.; Sonnleitner, B.; Lafferty, R. M. (1978) A rapid Gas Chromatographic Method for the Determination of Poly- $\beta$-Hydroxybutyric Acid in Microbial Biomass. Eur. J. Appl. Microbiol., 6, 29-37.

Carucci, A.; Dionisi, D.; Majone, M.; Rolle, E.; Smurra, P. (2001) Aerobic Storage by Activated Sludge on Real Wastewater. Water Res., 35, 3833-3844.

Choi, J.-i.; Lee, S. Y. (1997) Process Analysis and Economic Evaluation for Poly (3-hydroxybutyrate) Production by Fermentation. Bioprocess Eng., 17, 335-342. 
Coats, E. R.; Loge, F. J.; Smith, W. A.; Thompson, D. N.; Wolcott, M. P. (2007) Functional Stability of a Mixed Microbial Consortium Producing PHA from Waste Carbon Sources. Appl. Biochem. Biotechnol. (in press).

Coats, E. R.; Loge, F. J.; Wolcott, M. P.; Englund, K.; McDonald, A. G. (2007) Production of Natural Fiber Reinforced Thermoplastic Composites Through the Use of PHB-Rich Biomass. Bioresource Technol. (in press).

Comeau, Y.; Hall, K. J.; Hancock, R. E. W.; Oldham, W. K. (1986) Biochemical Model for Enhanced Biological Phosphorus Removal. Water Res., 20, 1511-1521.

Dionisi, D.; Majone, M.; Papa, V.; Beccari, M. (2004) Biodegradable Polymers from Organic Acids by Using Activated Sludge Enriched by Aerobic Periodic Feeding. Biotechnol. Bioeng., 85, 569-579.

Dionisi, D.; Renzi, V.; Majone, M.; Beccari, M.; Ramadori, R. (2004) Storage of Substrate Mixtures by Activated Sludges Under Dynamic Conditions in Anoxic or Aerobic Environments. Water Res., 38, 21962206.

Du, G.; Yu, J. (2002) Green Technology for Conversion of Food Scraps to Biodegradable Thermoplastic Polyhydroxyalkanoates. Environ. Sci. Technol., 36, 5511-5516.

Gerngross, T. U. (1999) Can Biotechnology Move Us Toward a Sustainable Society? Nat. Biotechnol., 17, 541-544.

Grady C. P. L.; Jr., Daigger, G. T.; Lim, H. C. (1999) Biological Wastewater Treatment, 2nd ed.; Marcel Dekker, Inc.: New York.

Hassan, M. A.; Nawata, O.; Shirai, Y.; Rahman, N. A. A.; Yee, P. L.; Bin Ariff, A.; Karim, M. I. A. (2002) A Proposal for Zero Emission from Palm Oil Industry Incorporating the Production of Polyhydroxyalkanoates from Palm Oil Mill Effluent. J. Chem. Eng. Jpn., 35, 9-14.

Kahar, P.; Tsuge, T.; Taguchi, K.; Doi, Y. (2004). High Yield Production of Polyhydroxyalkanoates from Soybean Oil by Ralstonia eutropha and its Recombinant Strain. Polym. Degrad. Stabil., 83, 79-86.

Lee, S. Y. (1996) Plastic Bacteria? Progress and Prospects for Polyhydroxyalkanoate Production in Bacteria. Trends Biotechnol., 14, 431438.

Lemoigne, M. (1926) Products of Dehydration and of Polymerization of B-Hydroxybutyric Acid. Bull. Soc. Chem. Biol. (Paris), 8, 770-782 (in French).
Madison, L. L.; Huisman, G. W. (1999) Metabolic Engineering of Poly(3hydroxyalkanoates): from DNA to Plastic. Microbiol. Mol. Biol. Rev., 63, 21-53.

Majone, M.; Ramadori, R.; Beccari, M. (1999) Biodegradable Polymers from Wastes by Using Activated Sludges Enriched by Aerobic Periodic Feeding. The Fourth Italian Conference on Chemical and Process Engineering, Florence, Italy, May 2-5, 667-670.

Marteel, A. E.; Davies, J. A.; Olson, W. W.; Abraham, M. A. (2003) Green Chemistry and Engineering: Drivers, Metrics, and Reduction to Practice. Ann. Rev. Environ. Res., 28, 401-428.

Mino, T.; Arun, V.; Tsuzuki, Y.; Matsuo, T. (1987) Biological Phosphate Removal from Wastewaters, Vol. 4, Ramador, R. (Ed.); Pergamon Press: Oxford, United Kingdom, 27-38.

Page, W. J.; Knosp, O. (1989) Hyperproduction of Poly- $\beta$-hydroxybutyrate During Exponential Growth of Azotobacter vinelandii UWD. Appl. Environ. Microbiol., 55, 1334-1339.

Randall, A. A.; Liu, Y.-H. (2002) Polyhydroxyalkanoates Form Potentially a Key Aspect of Aerobic Phosphorus Uptake in Enhanced Biological Phosphorus Removal. Water Res., 36, 3473-3478.

Reinsch, V. E.; Kelley, S. S. (1997) Crystallization of Poly(hydroxybutyrateco-hydroxyvalerate) in Wood Fiber-Reinforced Composites. J. Appl. Polym. Sci., 64, 1785-1796.

Reis, M. A. M.; Serafim, L. S.; Lemos, P. C.; Ramos, A. M.; Aguiar, F. R.; van Loosdrecht, M. C. M. (2003) Production of Polyhydroxyalkanoates by Mixed Microbial Cultures. Bioprocess Biosyst. Eng., 25, 377-385.

Shanks, R. A.; Hodzic, A.; Wong, S. (2004) Thermoplastic Biopolyester Natural Fiber Composites. J. Appl. Polym. Sci., 91, 2114-2121.

Takabatake, H.; Satoh, H.; Mino, T.; Matsuo, T. (2000) Recovery of Biodegradable Plastics from Activated Sludge Process. Water Sci. Technol., 42, 351-356.

Tchobanoglous, G.; Burton, F. L.; Stensel, H. D. (2003) Wastewater Engineering: Treatment and Reuse, 4th ed.; McGraw-Hill: New York.

Yamane, T. (1993) Yield of Poly-D(-)-3-hydroxybutyrate from Various Carbon Sources: A Theoretical Study. Biotechnol. Bioeng., 41, 165170.

Yu, J.; Si, Y. (2001) A Dynamic Study and Modeling of the Formation of Polyhydroxyalkanoates Combined with Treatment of High Strength Wastewater. Environ. Sci. Technol., 35, 3584-3588. 